\section{Reduction of burden of hemolyzed specimens in a large urban emergency department: A real-world, five years' experience}

\author{
Gianfranco Cervellin, ${ }^{1}$ Rosalia Aloe, ${ }^{2}$ \\ Laura Bonfanti, ${ }^{1}$ Patrizia Bonelli, ${ }^{2}$ \\ Giuseppe Lippi ${ }^{3}$ \\ 'Emergency Department, Academic \\ Hospital of Parma, Parma; ${ }^{2}$ Clinical \\ Chemistry Laboratory, Academic Hospital \\ of Parma, Parma; ${ }^{3}$ Section of Clinical \\ Biochemistry, University of Verona, \\ Verona, Italy
}

\begin{abstract}
In vitro hemolysis may jeopardize patient care because tests results generated using unsuitable specimens may lead to inappropriate patient management. The prevalence of hemolyzed specimens is high in the emergency department (ED). We previously showed that collecting blood by means of a closed system entailing manual aspiration of blood instead of using conventional evacuated systems was effective to cut-down by nearly half the rate of hemolysis. Aim of this real world study was to verify whether longterm replacement of standard evacuated blood collection systems may be really effective to reduce the burden of spurious hemolysis. Starting from May 2014 in the ED of our Hospital vacuum tubes were replaced with S-Monovette serum tubes. We compared data about hemolyzed specimens entered in the two years before the implementation of the new device (i.e., 2012 and 2013) and the two years after introducing SMonovette in manual aspiration mode (i.e. 2015 and 2016). The year 2014 was not considered due to mixed data. The rate of hemolyzed specimens decreased from $4.36 \%$ to $3.07 \%$ with the use of S-Monovette in manual aspiration mode (Chi squared, 183.8; $\mathrm{P}<0.001)$. The likelihood of obtaining hemolyzed specimens was hence reduced by approximately 30\% (relative risk, 0.707), with an expected economic saving of approximately $510 € /$ year. The results of this real-world study demonstrate that the use of an alternative closed device encompassing manual aspiration for drawing blood from intravenous catheters may reduce hemolyzed samples by approximately $30 \%$, so representing a valuable perspective for safeguarding patient safety and improving ED efficiency.
\end{abstract}

\section{Introduction}

The term hemolysis (or haemolysis) originates from the Greek hemo (i.e., blood), and lysis (i.e., breakdown). Hemolysis is a pathological process encompassing the breakdown of red blood cells (RBCs), so resulting in massive release of hemoglobin and other intracellular compounds in plasma. ${ }^{1}$ In clinical medicine, distinguishing in vivo and in vitro hemolysis is of outmost importance, since the former may be due to a number of conditions and disorders, whereas the latter is substantially attributable to problems occurred during collection, handling or transportation of blood specimens. In vitro hemolysis may seriously jeopardize patient care because tests results generated on unsuitable specimens may derange the diagnostic reasoning and lead to inappropriate patient management. ${ }^{1}$ The prevalence of hemolyzed specimens has been found especially high in healthcare settings entailing blood collection from intravenous lines. ${ }^{2}$ These conventionally include the emergency department (ED), intensive care unit (ICU), as well as neonatal and pediatric wards. In the ED, in particular, the risk of collecting hemolyzed specimens is magnified, with a rate of unsuitable specimens that can even approximate $12 \%$ of all collected samples. ${ }^{2}$

Such a high prevalence of hemolyzed specimens collected in the ED has led to propose many potential strategies, but none of these was proven to be really effective to solve all the potential issues. ${ }^{3,4}$

In an observational study conducted in a large urban ED we previously showed that collecting blood by means of a closed system entailing manual aspiration of blood instead of using conventional evacuated systems was effective to cut-down by nearly half the rate of hemolysis (i.e., from $4.41 \%$ to $2.57 \%$ ). ${ }^{5}$ Therefore, the aim of this observational, real world, study was to verify whether long-term replacement of standard evacuated blood collection systems with closed system entailing manual aspiration of blood (SMonovette, Sarstedt AG \& Co., Nümbrecht, Germany) may be really effective to reduce the burden of spurious hemolysis.

\section{Materials and Methods}

This study entailed the systemic identification of hemolysis in all serum samples received in the clinical laboratory of the University Hospital of Parma from the local ED, a large urban facility with approximately 94.000 visits per years. In the local ED, blood samples are drawn through intravenous catheters $(1.0 \times 3.2 \mathrm{~mm}, 20$-gauge catheter;
Correspondence: Gianfranco Cervellin, Emergency Department, Academic Hospital of Parma, Via Gramsci 14, 43126 Parma, Italy. Tel: +390521703800

E-mail: gianfranco.cervellin@gmail.com; gcervellin@ao.pr.it

Key words: hemolysis; hemolyzed specimens; preanalytical variability; blood collection tube; blood drawing.

Received for publication: 15 May 2017. Revision received: 26 June 2017. Accepted for publication: 27 June 2017.

This work is licensed under a Creative Commons Attribution 4.0 License (by-nc 4.0).

CC Copyright G. Cervellin et al., 2017

Licensee PAGEPress, Italy

Emergency Care Journal 2017; 13:6793

doi:10.4081/ecj.2017.6793

Neo DELTA VEN, Viadana, MN, Italy). Starting from May $15^{\text {th }}, 2014$, the Direction of the University Hospital authorized the replacement of standard $13 \times 100 \mathrm{~mm} \times 5.0$ $\mathrm{mL}$ BD Vacutainer ${ }^{\circledR}$ SST II Plus plastic serum tubes (Becton Dickinson Italia S.p.A, Milan, Italy) with $4.0 \mathrm{~mL}$ S-Monovette serum tubes. After an appropriate period of training, starting from June $1^{\text {st }} 2014$ blood started to be collected only using S-Monovette blood tubes in aspiration mode. The detailed description of this device has been provided elsewhere. ${ }^{5}$ In the local laboratory, all serum samples are systematically screened for hemolysis after centrifugation by photometrical assessment on Beckman Coulter AU5800 (Beckman Coulter Inc., Brea, CA, USA). The analyzer provides a semi-quantitative measure of hemolysis from ( 0 to $5 \mathrm{~g} / \mathrm{L})$ as hemolysis index (HI) by means of bichromatic readings at $410 / 480$ and $600 / 800 \mathrm{~nm}$. As for local practice, established in accordance with current indication, ${ }^{6}$ hemolysis is considered significant (i.e., generating a bias in the measurement of hemolysis-sensitive analytes such as potassium and lactate dehydrogenase) when the HI is $\geq 0.5 \mathrm{~g} / \mathrm{L}$.

The information of all hemolyzed specimens received in the local laboratory are systematically entered into a specific software, designed for recording a vast array of preanalytical problems. ${ }^{7}$ The analysis was based on data about hemolyzed specimens entered in the two years before the implementation of the new blood drawing device (i.e., 2012 and 2013) and the two years after introducing S-Monovette in manual aspiration mode (i.e. 2015 and 2016). The year 2014 was not considered in our analysis due to mixed data between one system and the other and the period of training necessary to the nurses to 
get used to the new system. The statistical analysis was based on Chi squared statistics with Yates's correction and relative risk estimation (with 95\% confidence interval; 95\% CI), using Analyse-it (Analyse-it Software Ltd, Leeds, UK) and MedCalc Version 12.3.0 (MedCalc Software, Mariakerke, Belgium). The study was carried out in accordance with the Declaration of Helsinki, under the terms of all relevant local legislations and was cleared by the Institutional Review Board.

\section{Results}

The main results of this study are shown in Figure 1. Despite a constant increase in the number of specimens collected in the ED was observed, mostly due to the parallel increase in the number of $\mathrm{ED}$ visits from 2012 to 2016, the rate of hemolyzed specimens significantly decreased, from $4.36 \%$ before the introduction of the new system to $3.07 \%$ with the use of S-Monovette in manual aspiration mode (Chi squared, 183.8; $\mathrm{P}<0.001)$. The likelihood of obtaining hemolyzed specimens was hence reduced by approximately $30 \%$ (relative risk, 0.707; 95\% CI, 0.672-0.743; $\mathrm{P}<0.001)$ after the introduction of the new system. As regards the potential economic savings, we have estimated that the much lower burden of hemolyzed samples occurring after the introduction of S-Monovette would have allowed saving approximately 1073 blood tubes (including double recollections) over the total amount of those collected in the years 2012-2013. As for national regulation, replacement of a medical device within an ongoing tender is only possible if the new device is offered at the same price as, or at a lower cost than, the former. Therefore, considering the actual cost of a single blood tube (i.e., $0.46 €$ ), the cost of a new blood tube label (i.e., $0.05 €$ ), the estimated cost attributable to nurses drawing an additional blood tube (i.e., $\sim 0.22 € / \mathrm{min}$ ), the introduction of the new blood tubes would translate into an expected economic saving of approximately $510 € /$ year. Since repeated sampling is always associated with diagnostic delay and prolonged stay in the ED (usually between, 2 to 3 hours per patient, according to the type of testing), we can also suppose that between 990-1485 patient-hours have been gained in the local ED.

\section{Discussion}

The collection of hemolyzed specimens remains an important problem in modern medicine. This is mostly attributable to the potential impact on patient safety, of diagnostic delays caused by recollection of additional specimens, or to the derangement of managed care when medical decisions are taken on unreliable data generated from the analysis of unsuitable specimens. In vitro hemolysis is also associated with organizational and economic issues. Sample recollection necessitates the use of another blood tube, another tube label and, frequently, another needle when blood is drawn with standard venipuncture, but also entails a potentially avoidable activity (e.g., sample recollection may consume 1 to 3 min of phlebotomist's time). The need of recollecting unsuitable samples also contributes to

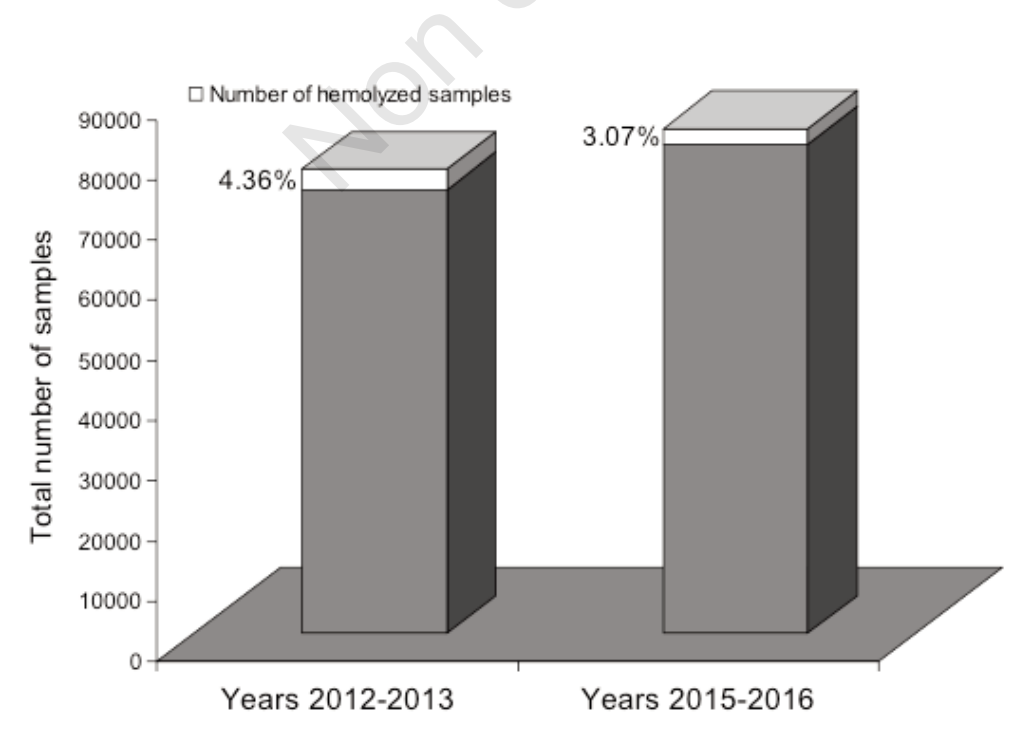

Figure 1. Hemolysis rate in serum samples collected from intravenous lines in a large emergency department using a conventional evacuated system (years 2012-2013) and with the S-Monovette system in aspiration mode (years 2015-2016).

increase overcrowding in short stay units such as the ED, since the patients cannot be discharged or hospitalized until complete laboratory data are available to the emergency physician.

\section{Conclusions}

The results of this real-world study, based in a large urban ED, convincingly demonstrate that replacing standard evacuated blood tubes with an alternative closed device encompassing manual aspiration when drawing blood from intravenous catheters may reduce the need to recollect hemolyzed samples by approximately $30 \%$, so representing a valuable perspective for safeguarding patient safety and improving efficiency both in the ED and in the laboratory. Unfortunately, no systematic survey has been carried out to assess the level of compliance of the procedure and/or the satisfaction of the nursing staff. This may be indeed a reasonable topic for a further study.

\section{References}

1. Lippi G, Plebani M, Di Somma S, Cervellin G. Hemolyzed specimens: a major challenge for emergency departments and clinical laboratories. Crit Rev Clin Lab Sci 2011;48:143-53.

2. Lippi G, Cervellin G, Mattiuzzi C. Critical review and meta-analysis of spurious hemolysis in blood samples collected from intravenous catheters. Biochem Med (Zagreb) 2013;23:193200.

3. Kavsak PA. A step closer in reducing hemolysis in blood samples collected in the emergency department. Clin Biochem 2013;46:565.

4. Dietrich H. One poke or two: can intravenous catheters provide an acceptable blood sample? A data set presentation, review of previous data sets, and discussion. J Emerg Nurs 2014;40:575-8.

5. Lippi G, Bonelli P, Bonfanti L, Cervellin G. The use of S-Monovette is effective to reduce the burden of hemolysis in a large urban emergency department. Biochem Med (Zagreb) 2015;25:69-72.

6. Simundic AM, Topic E, Nikolac N, Lippi $\mathrm{G}$. Hemolysis detection and management of hemolysed specimens. Biochem Med 2010;20:154-9.

7. Lippi G, Bonelli P, Rossi R, et al. Development of a preanalytical errors recording software. Biochem Med (Zagreb) 2010;20:90-5. 